\title{
Measure theoretic entropy of the system of hard spheres
}

\author{
MACIEJ P. WOJTKOWSKI \\ Department of Mathematics, University of Arizona, Tucson, AZ 85721, USA
}

(Received 20 May 1986 and revised 12 January 1987)

\begin{abstract}
We estimate from below the measure theoretic entropy of the system of spheres interacting by elastic collisions. We find the asymptotics of the entropy in the case of two disks on atoms as the radius increases. We establish that high density leads to large entropy. We introduce a general estimate for Lyapunov exponents in the case of special symplectic matrices.
\end{abstract}

\section{Introduction}

Let us consider the system of $N$ spheres of radius $r$ in a volume $\Lambda$ interacting by elastic collisions. We fix the total energy of the system to be $N E$. The dynamics is described by a measurable flow $\Phi^{t}$ preserving the microcanonical probability distribution $\nu$. The measure theoretic entropy of the flow $h\left(\Phi^{1}\right)$ was shown by Sinai ([Sin $3]$, [Cher]) to be positive. One may ask what is the connection between this quantity and the equilibrium statistical mechanics of the system. In this direction Sinai and Chernov ([Sin 2], [Cher-Sin]) showed that the thermodynamic limit of $h\left(\Phi^{1}\right)$ exists for small densities and is connected with the entropy of the infinite system with respect to the group of time-space translations. More precisely

$$
\lim \frac{1}{N} h\left(\Phi^{1}\right)=h(\rho, E),
$$

where the limit is taken as $\Lambda \rightarrow \mathbb{R}^{3}$ and the density $N /$ volume $(\Lambda) \rightarrow \rho$. Suppose that the limit exists for all densities. Then we conjecture that

$$
\lim _{\rho \rightarrow \rho_{\max }} h(\rho, E)=+\infty .
$$

What we actually establish rigorously is the following estimate when $\Lambda$ is a torus

$$
h\left(\Phi^{1}\right) \geq \int \tau^{-1} \sinh ^{-1} \sqrt{\tau\left|V_{\text {rel }}\right|(r \cos \psi)^{-1}} d \nu,
$$

where $\tau$ is the time between the nearest collision in the past and the nearest collision in the future, $V_{\mathrm{rel}}$ is the relative velocity of the colliding spheres and $\psi$ is the angle between $V_{\text {rel }}$ and the line through the centers of the colliding spheres, both quantities correspond to the nearest future collision.

Since it is reasonable to believe that $\tau \sim 1 / N$ the estimate does not look impressive because it yields only a quantity $\sim \sqrt{N}$ which is not enough even to establish the 
positivity of the entropy per particle $h(\rho, E)$. But if we keep the volume $\Lambda$ and the number of spheres fixed a.ld increase the effective density by increasing the radius $r$ then we can expect the average time between collisions to go to zero and since our estimate is roughly $\int \tau^{-1 / 2} d \nu$ then $h\left(\Phi^{1}\right) \rightarrow+\infty$. If we accept that $h\left(\Phi^{1}\right)$ is essentially an additive function of the volume (which is the basis of the existence of the thermodynamic limit) we must conclude that $h(\rho, E) \rightarrow+\infty$ when $\rho \rightarrow \rho_{\max }$.

Actually we find the mathematics of dense packing so unwieldy that we are unable to show rigorously that the integral in our estimate indeed goes to infinity when $r$ approaches its maximal value. We supply only heuristic arguments.

The meaning of the entropy per particle being large for an almost dense packing of spheres is open to interpretation. One possibility is that this quantity is not relevant to the statistical mechanics of the dense system.

The plan of the paper is the following. In $\S 1$ we consider two disks on a torus. Following Sinai [Sin 1] we reduce the system to the billiard system in a plane domain which for large disks is a unit square with the sides replaced by arcs of circles with small curvature $\varepsilon$. Benettin [Ben] established numerically that the asymptotics of the measure theoretic entropy (Lyapunov exponent) of this billiard system is const $\cdot \sqrt{\varepsilon}$ as $\varepsilon \rightarrow 0$. We prove this rigorously and even estimate the constant to be between 1.91 and 2 . This asymptotics is sufficient to cause the entropy of the system of two disks to go to $+\infty$ when the radius $r \rightarrow r_{\max }$. Note that this fact is not something one can easily see qualitatively. If for example the asymptotics in the billiard problem were const - $\varepsilon$ then the entropy in the system of disks would stay bounded. The trade off is the following: when the disks are large there are many collisions per unit time but when the time between collisions is small the contribution from each collision into the entropy is also small. The net effect of making the disks large is to be computed.

The method we use for the lower bound was developed in [Wojt 2] and for the upper bound was inspired by a conversation with A. Katok. In the rest of the paper we generalize the lower bound to the multidimensional case. In $\$ \S 2$ and 3 we develop a general method of estimating from below Lyapunov exponents in the symplectic case. These sections are independent of the rest of the paper. We develop there the discrete time version of the method of estimating the measure theoretic entropy of the geodesic flow in the case of nonpositive curvature from [Ball-Wojt]. The generality of exposition here exceeds the needs of the application to the gas of hard spheres in $\S 4$ but we hope that in such a general setting the method may prove useful elsewhere. In $\S 2$ we discuss $Q$-monotone symplectic operators - they were essentially introduced in [Wojt 1]. In Appendices A and B we supply some more technical computations for $\S \S 1$ and 4 respectively.

\section{Two hard disks on a torus}

Let us consider the system of two hard disks of unit mass on a torus $\mathbb{T}^{2}=\mathbb{R}^{2} / \mathbb{Z}^{2}$. The torus is flat with unit 'sides' so that the radius of the disk $r<\sqrt{2} / 4$. The disks move uniformly until collision and they collide elastically. 
By $q_{i} \in \mathbb{R}^{2}, q_{i} \bmod 1, i=1,2$ we denote the position of the centers of the disks. The configuration space $\tilde{K}$ of our system is thus

$$
\tilde{K}=\left\{\left(q_{1}, q_{2}\right) \bmod 1|| q_{2}-q_{1} \mid \geq 2 r\right\} .
$$

We introduce new coordinates $\left(u_{1}, u_{2}\right) \bmod 1$ in $\tilde{K}$ by the formula $q_{1}=u_{1}-u_{2}$, $q_{2}=u_{1}+u_{2}$. The mapping $\left(u_{1}, u_{2}\right) \bmod 1 \rightarrow\left(q_{1}, q_{2}\right) \bmod 1$ is a 4 to 1 covering. The preimage of $\tilde{K}$ under this mapping is

$$
K=\left\{\left(u_{1}, u_{2}\right) \bmod 1|| u_{2}-\frac{1}{2} z \mid \geq r \text { for any } z \in \mathbb{Z}^{2}\right\} .
$$

Hence $K$ is the Cartesian product of $\mathbb{T}^{2}$ and the torus with four disks of radius $r$ removed, which we denote by $\pi_{r}^{2}$.

The phase space of our system is the tangent bundle of $K$ with obvious identifications at the boundary. The total energy

$$
E=\frac{1}{2}\left(\dot{q}_{1}^{2}+\dot{q}_{2}^{2}\right)=\dot{u}_{1}^{2}+\dot{u}_{2}^{2}
$$

and the total momentum $\dot{u}_{1}$ are first integrals of the system.

The flow describing the dynamics in the phase space preserves the Lebesgue measure. Fixing the total energy $E$ we obtain a flow preserving a finite smooth measure. We are interested in the metric entropy $H=H(E, r)$ of the flow.

With the total energy fixed the total momentum $\dot{u}_{1}$ can assume values from a disk of radius $\sqrt{E}$. The uniform measure on the sphere $\dot{u}_{1}^{2}+\dot{u}_{2}^{2}=E$ projects onto the uniform measure on the disk $\dot{\boldsymbol{u}}_{1}^{2} \leq E$.

It is not difficult to see that the flow for every fixed $\dot{u}_{1}$ is the Cartesian product of the quasi-periodic flow on $\mathbb{T}^{2}$ with the velocity $\dot{u}_{1}$ and the billiard flow in $\mathbb{\nabla}_{r}^{2}$ with the speed $l=\sqrt{E-\dot{u}_{1}^{2}}$. The phase space for the billiard flow is $S_{l} \pi_{\mathrm{r}}^{2}$ - the circle bundle over $\mathbb{T}_{r}^{2}$ of tangent vectors of length $l=\sqrt{E-\dot{u}_{1}^{2}}$.

Let $h(r)$ denote the metric entropy of the billiard flow in $S_{1} \pi_{r}^{2}$ (i.e. with the speed 1). The entropy of the billiard flow in $S_{l} \mathbb{T}_{r}^{2}$ is then equal to $l h(r)$.

Since the entropy of the quasi-periodic flow is zero, then the entropy $H(E, r)$ is the average value of $\sqrt{E-\dot{u}_{1}^{2}} h(r)$ with respect to the uniform measure on the disk $\dot{u}_{1}^{2} \leq E$. Hence we get

$$
H(E, r)=\frac{2 \pi h(r) \int_{0}^{\sqrt{E}} \sqrt{E-p^{2}} p d p}{\pi E}=\frac{2}{3} \sqrt{E} h(r)
$$

Our goal is to find the asymptotics of $h(r)$ as $r \rightarrow \sqrt{2} / 4$.

When $r>\frac{1}{4}, \mathbb{T}^{2}$ has four connected components, each equal to a plane domain bounded by four arcs of circles of radius $r$ (see figure 1(a)). The entropy $h(r)$ is equal to the metric entropy of the billiard flow in such a domain. We rescale the domain so that we get the domain $Q_{\varepsilon}$ inscribed in the unit square $Q_{0}$ (see figure $1(b))$. The curvature of the boundaries is

$$
\varepsilon=\frac{\sqrt{2}}{4} \frac{1-\sqrt{16 r^{2}-1}}{r} .
$$

Let $g(\varepsilon)$ be the entropy of the billiard flow in $Q_{\varepsilon}$. Since the change of scale amounts 
to the change in velocity we obtain

$$
g(\varepsilon)=h(r) \varepsilon r
$$

We introduce a new parameter $\eta=((\sqrt{2} / 4)-r) /(\sqrt{2} / 4)$. Then $\varepsilon=2 \eta+O\left(\eta^{2}\right)$ and $\eta \rightarrow 0$ as $r \rightarrow \sqrt{2} / 4$.

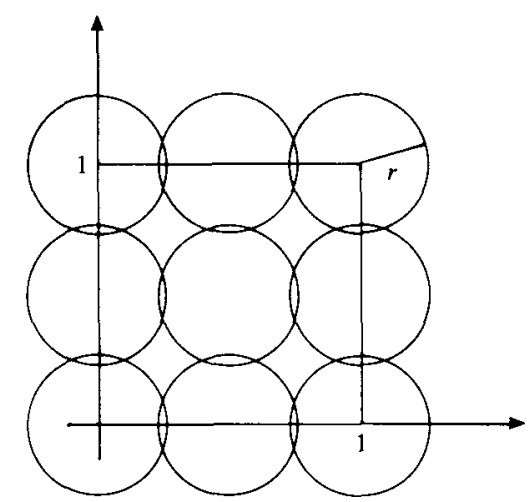

Figure 1(a)

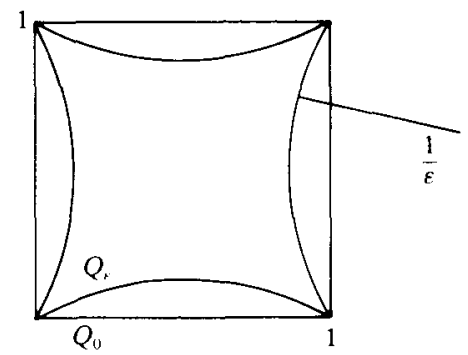

Figure 1(b)

THEOREM 1.

$$
\liminf _{\varepsilon \rightarrow 0} g(\varepsilon) / \sqrt{\varepsilon} \geq \alpha_{0}, \quad \limsup _{\varepsilon \rightarrow 0} g(\varepsilon) / \sqrt{\varepsilon} \leq 2
$$

where

$$
\alpha_{0}=\sqrt{2}+\frac{4}{3}-\pi^{-1}\left(\frac{2 \sqrt{2}}{3} \ln 2+\frac{4}{3} \ln (3+\sqrt{2})\right) \approx 1.909 .
$$

We conjecture that actually

$$
\lim _{\varepsilon \rightarrow 0} \frac{g(\varepsilon)}{\sqrt{\varepsilon}}=2 .
$$

This would imply that $h(r) \approx 4 / \sqrt{\eta}$ or $H(E, r) \approx \frac{8}{3} \sqrt{E}(1 / \sqrt{\eta})$ when $r \rightarrow \sqrt{2} / 4$.

Theorem 1 is sufficient to conclude that $\lim _{r \rightarrow \sqrt{2} / 4} H(E, r)=+\infty$.

Proof. Let us consider the billiard system in $Q_{E}$. The phase space of the system is the unit tangent bundle of $Q_{\varepsilon}$ with appropriate identifications at the boundary. As usual we replace the original billiard flow by the standard section map. Let $\Sigma_{\varepsilon}$ be the space of unit tangent vectors attached at the boundary of $Q_{\varepsilon}$ and pointing inwards. We introduce coordinates $(s, \phi)$ in $\Sigma_{\varepsilon}$ where $s$ is the arc length parameter along the boundary and $\phi, 0 \leq \phi \leq \pi$ is the angle which the tangent vector makes with the boundary. The standard section map $T: \Sigma_{\varepsilon} \rightarrow \Sigma_{\varepsilon}$ is the map of the first return to $\Sigma_{\varepsilon}$. The natural invariant measure for $T$ is

$$
d \mu=\text { const } \cdot \sin \phi \cdot d s d \phi .
$$

Let $\tau: \Sigma_{\varepsilon} \rightarrow \mathbb{R}^{+}$be the time of the first return i.e. the time till the next reflection at the boundary. 
To describe the differential $D T$ we use special linear coordinates $(d u, d v)$ in tangent planes of $\Sigma_{\varepsilon}$ given by the formulas

$$
\begin{aligned}
d u & =\sin \phi d s, \\
d v & =\varepsilon d s-d \phi .
\end{aligned}
$$

(These coordinates come from the natural coordinates in the plane orthogonal to the orbit of our flow.) In these coordinates the matrix of $D T$ at $x=(s, \phi)$ has the form

$$
\left|\begin{array}{cc}
1 & \tau \\
\frac{2 \varepsilon}{\sin \phi_{1}} & 1+\frac{2 \varepsilon \tau}{\sin \phi_{1}}
\end{array}\right|
$$

where $T(s, \phi)=\left(s_{1}, \phi_{1}\right)$. In Appendix B we derive this formula in the multidimensional setting.

Let $\lambda(x)=\lim _{n \rightarrow+\infty} 1 / n \ln \left\|D_{x} T^{n}\right\|, x \in \Sigma_{\varepsilon}$ be the nonnegative Lyapunov exponent of $T .(\lambda(x)$ is actually constant almost everywhere by the ergodicity of $T, \mathrm{cf}$. [Sin 1], [Bun-Sin] but our discussion does not depend on this subtle fact.)

Since the matrices $D T$ have all positive entries they map the positive quadrant into itself and we can use theorem 2 from [Wojt 2] which yields

$$
\int_{\Sigma_{\varepsilon}} \lambda(x) d \mu \geq \int_{\Sigma_{\varepsilon}} \ln \left(\sqrt{1+\frac{2 \varepsilon \tau}{\sin \phi_{1}}}+\sqrt{\frac{2 \varepsilon \tau}{\sin \phi_{1}}}\right) d \mu=\int_{\Sigma_{\varepsilon}} \sinh ^{-1} \sqrt{\frac{2 \varepsilon \tau}{\sin \phi_{1}}} d \mu .
$$

This estimate is also a special case of the multidimensional estimate which we will prove later (see theorem 4 ).

$T$ is not continuous but its singularities are mild enough for the Pesin formula to hold, cf. [Pes], [Led-Str], [Kat-Str]. Actually for the billiard system an equivalent formula was proved by Sinai [Sin 1]. By the Pesin formula the metric entropy of $T$ is equal to $\int_{\Sigma} \lambda(x) d \mu$. Further, by the Abramov formula, the metric entropy $g(\varepsilon)$ of the time one map of the flow is equal to

$$
g(\varepsilon)=\int_{\Sigma_{\varepsilon}} \lambda(x) d \mu / \int_{\Sigma_{\varepsilon}} \tau(x) d \mu .
$$

By the Lebesgue Dominated Convergence Theorem

$$
\begin{aligned}
\liminf _{\varepsilon \rightarrow 0} \frac{g(\varepsilon)}{\sqrt{\varepsilon}} & \geq \lim _{\varepsilon \rightarrow 0} \int_{\Sigma_{\varepsilon}} \frac{\sinh ^{-1} \sqrt{\frac{2 \varepsilon \tau}{\sin \phi_{1}}}}{\sqrt{\varepsilon}} \mathrm{d} \mu / \lim _{\varepsilon \rightarrow 0} \int_{\Sigma_{\varepsilon}} \tau d \mu \\
& =\int_{\Sigma_{0}} \sqrt{\frac{2 \tau}{\sin \phi_{1}}} d \mu / \int_{\Sigma_{0}} \tau d \mu,
\end{aligned}
$$

where the last integrals are taken in the square $Q_{0}$. It turns out that these integrals can be computed explicitly and the result is equal to $\alpha_{0}$. We do it in Appendix A.

To estimate the entropy from above we will use the following obvious fact

$$
\int_{\Sigma_{\varepsilon}} \lambda(x) d \mu \leq \int_{\Sigma_{\varepsilon}} \ln \|D T\| d \mu .
$$


But we will once again change coordinates in the tangent planes to $\Sigma_{\varepsilon}$ :

$$
\begin{aligned}
& d \tilde{u}=(a \varepsilon)^{\frac{1}{4}} d u \\
& d \tilde{v}=(a \varepsilon)^{-\frac{1}{4}} d v
\end{aligned}
$$

where $a$ is a constant to be chosen later. In these new coordinates $D T$ has the form

$$
\left|\begin{array}{cc}
1 & \sqrt{a} \sqrt{\varepsilon} \tau \\
\frac{2 \sqrt{\varepsilon}}{\sqrt{a} \sin \phi_{1}} & 1+\frac{2 \varepsilon \tau}{\sin \phi_{1}}
\end{array}\right|
$$

By straightforward computations we obtain

where

$$
\|D T\|=\sqrt{1+\frac{\Delta}{2}+\sqrt{\Delta} \sqrt{1+\frac{\Delta}{4}}},
$$

$$
\Delta=\varepsilon\left(\sqrt{a} \tau+\frac{2}{\sqrt{a} \sin \phi_{1}}\right)^{2}+\frac{4 \varepsilon^{2} \tau^{2}}{\sin ^{2} \phi_{1}} .
$$

By the Lebesgue Dominated Convergence Theorem,

$$
\begin{aligned}
\limsup _{\varepsilon \rightarrow 0} \frac{g(\varepsilon)}{\sqrt{\varepsilon}} & \leq \lim _{\varepsilon \rightarrow 0} \int_{\Sigma_{\varepsilon}} \frac{1}{\sqrt{\varepsilon}} \ln \|D T\| d \mu / \lim _{\varepsilon \rightarrow 0} \int_{\Sigma_{\varepsilon}} \tau d \mu \\
& =\frac{1}{2} \int_{\Sigma_{0}}\left(\sqrt{a} \tau+\frac{2}{\sqrt{a} \sin \phi_{1}}\right) d \mu / \int_{\Sigma_{0}} \tau d \mu .
\end{aligned}
$$

Now we choose the constant $a$ so that the last quantity has the minimal value i.e.

We get

$$
a=\int_{\Sigma_{0}} \frac{2}{\sin \phi_{1}} d \mu / \int_{\Sigma_{0}} \tau d \mu .
$$

$$
\limsup _{\varepsilon \rightarrow 0} \frac{g(\varepsilon)}{\sqrt{\varepsilon}} \leq \sqrt{\int_{\Sigma_{0}} \frac{2}{\sin \phi_{1}} d \mu / \int_{\Sigma_{0}} \tau d \mu .}
$$

The integrals on the right-hand side are taken explicitly in Appendix $A$ and the result is equal to 2 .

\section{Q-monotone symplectic operators}

Let $V$ be an $n$-dimensional euclidean space with the scalar product $\langle\cdot, \cdot\rangle$. We introduce the standard symplectic structure in $\mathbb{V} \times \mathbb{V}$,

$$
\omega\left(v_{1}, v_{2}\right)=\left\langle x_{1}, y_{2}\right\rangle-\left\langle x_{2}, y_{1}\right\rangle
$$

where $v_{i}=\left(x_{i}, y_{i}\right) \in \mathbb{V} \times \mathbb{V}, i=1,2$.

Let $\operatorname{Sp}(\mathbb{V})$ be the group of linear symplectic operators on $\mathbb{V} \times \mathbb{V}$. A symplectic operator $S$ on $\mathbb{V} \times \mathbb{V}$ can be described by a $2 \times 2$ matrix of linear operators on $\mathbb{V}$.

If we put

$$
S=\left(\begin{array}{ll}
A & B \\
C & D
\end{array}\right)
$$

$$
J=\left(\begin{array}{rr}
0 & -I \\
I & 0
\end{array}\right)
$$


where $I$ is the identity operator on $\mathbb{V}$ then the symplecticity of $S$ is equivalent to $S^{*} J S=J$ i.e.

$$
C^{*} A=A^{*} C, \quad D^{*} B=B^{*} D \quad \text { and } \quad A^{*} D-C^{*} B=I .
$$

We consider additionally the quadratic form $Q$ on $\vee \times V$

$$
Q(v)=\langle x, y\rangle \quad \text { where } v=(x, y) \in \mathbb{V} \times \mathbb{V}
$$

and the 'cone' $\mathbb{C}=\{v \in \mathbb{V} \times \mathbb{V} \mid Q(v) \geq 0\}$.

Definition. A linear operator $S$ is $Q$-monotone if $Q(S v) \geq Q(v)$ for every $v \in \mathbb{V} \times \mathbb{V}$.

THEOREM 2. Let

$$
S=\left(\begin{array}{ll}
A & B \\
C & D
\end{array}\right)
$$

be a linear symplectic operator on $\mathbb{V} \times \mathbb{V}$. Then the following are equivalent:
(a) $S$ is Q-monotone;
(b) $S \mathbb{C} \subset \mathbb{C}$;
(c) $A$ is invertible and $B A^{*} \geq 0$ and $A^{*} C \geq 0$;
(d) $D$ is invertible and $C D^{*} \geq 0$ and $D^{*} B \geq 0$.

Proof. (a) $\Rightarrow$ (b) is obvious.

(b) $\Rightarrow$ (c) First we will prove that $A$ must be invertible. Suppose that there is $x_{0} \in \mathbb{V}$ such that $A x=0$. By symplecticity

so that $B^{*} C x_{0}=-x_{0}$.

$$
D^{*} A-B^{*} C=I
$$

Let $v=\left(x_{0}, y\right) \in \mathbb{C}$ i.e. $\left\langle x_{0}, y\right\rangle \geq 0$, then

$$
S v=\left(B y, C x_{0}+D y\right) \in \mathbb{C} \text {. }
$$

Hence we get

$$
0 \leq\left\langle B y, C x_{0}+D y\right\rangle=-\left\langle x_{0}, y\right\rangle+\left\langle D^{*} B y, y\right\rangle
$$

or

$$
\left\langle x_{0}, y\right\rangle \leq\left\langle D^{*} B y, y\right\rangle .
$$

Putting $y=\varepsilon x_{0}, \varepsilon \geq 0$ we obtain

$$
\left\langle x_{0}, x_{0}\right\rangle \leq \varepsilon\left\langle D^{*} B x_{0}, x_{0}\right\rangle
$$

for all $\varepsilon \geq 0$, so that $x_{0}=0$.

The operator

$$
\left(\begin{array}{cc}
A^{-1} & 0 \\
0 & A^{*}
\end{array}\right)
$$

is symplectic and preserves $Q$ so that if

$$
S_{1}=\left(\begin{array}{cc}
A^{-1} & 0 \\
0 & A^{*}
\end{array}\right)\left(\begin{array}{cc}
A & B \\
C & D
\end{array}\right)=\left(\begin{array}{cc}
I & A^{-1} B \\
A^{*} C & A^{*} D
\end{array}\right)
$$

then $S_{1} \mathbb{C}=S \mathbb{C} \subset \mathbb{C}$.

Let us put $R=A^{-1} B, P=A^{*} C$. We have

$$
S_{1}=\left(\begin{array}{cc}
I & R \\
P & I+P^{*} R
\end{array}\right)
$$


and since $S_{\mathrm{t}}$ is symplectic, then, by (1), $P$ and $R$ must be self adjoint. If $Q(v)=\langle x, y\rangle \geq 0$ then

$$
0 \leq Q(S v)=Q\left(S_{1} v\right)=\langle x, y\rangle+\langle R y, y\rangle+\langle P(x+R y), x+R y\rangle .
$$

Letting $y=0$ we get that $P=A^{*} C \geq 0$.

If $R$ is not positive semidefinite then there is $y_{0}$ such that $\left\langle R y_{0}, y_{0}\right\rangle<0$. Let $x_{0}$ be such that $\left\langle x_{0}, y_{0}\right\rangle<0$. Consider $v=\left(\varepsilon x_{0}-R y_{0}, y_{0}\right)$. We have

$$
Q(v)=\varepsilon\left\langle x_{0}, y_{0}\right\rangle-\left\langle R y_{0}, y_{0}\right\rangle \geq 0
$$

for sufficiently small $\varepsilon>0$ and then also $Q\left(S_{1} v\right) \geq 0$. But

$$
Q\left(S_{1} v\right)=\varepsilon\left\langle x_{0}, y_{0}\right\rangle+\varepsilon^{2}\left\langle P x_{0}, x_{0}\right\rangle<0
$$

for sufficiently small $\varepsilon>0$. The obtained contradiction proves that $R \geq 0$. Finally $B A^{*}=A R A^{*} \geq 0$.

(c) $\Rightarrow$ (a) Using the above notation

$$
Q(S v)=Q\left(S_{1} v\right)=\langle x, y\rangle+\langle R y, y\rangle+\langle P(x+R y), x+R y\rangle \geq\langle x, y\rangle=Q(v)
$$

since $P=A^{*} C \geq 0$ and $R=A^{-1}\left(B A^{*}\right) A^{-1 *} \geq 0$.

In a similar way one can prove that $(\mathrm{b}) \Rightarrow(\mathrm{d})$ and $(\mathrm{d}) \Rightarrow$ (a).

LEMMA 1. If $P \geq 0$ and $R \geq 0$ are self-adjoint positive semidefinite operators on $\mathbb{V}$ then $P R$ has only real nonnegative eigenvalues.

Proof. If one of the operators e.g. $P$ is positive definite then $P R$ is similar to

$$
P^{-\frac{1}{2}} P R P^{\frac{1}{2}}=P^{\frac{1}{2}} R P^{\frac{1}{2}}
$$

which is self-adjoint and positive semidefinite. The general case follows from the continuity of the spectrum.

Corollary 1. If

$$
S=\left(\begin{array}{ll}
A & B \\
C & D
\end{array}\right)
$$

is symplectic and $Q$-monotone then $C^{*} B$ has only real nonnegative eigenvalues.

Proof. By Theorem 2, $B A^{*} \geq 0$ and $C^{*} A \geq 0$. Hence also $A^{-1} B=A^{-1}\left(B A^{*}\right) A^{-1 *} \geq 0$. Now we can apply Lemma 1 to $C^{*} A$ and $A^{-1} B$.

For a symplectic $Q$-monotone operator

$$
S=\left(\begin{array}{ll}
A & B \\
C & D
\end{array}\right)
$$

let $u_{1} \geq u_{2} \geq \cdots \geq u_{n} \geq 0$ be the eigenvalues of $C^{*} B$. We define

$$
\rho(S)=\prod_{i=1}^{n}\left(\sqrt{1+u_{i}}+\sqrt{u_{i}}\right)=\exp \left(\sum_{i=1}^{n} \sinh ^{-1} \sqrt{u_{i}}\right)
$$

Let $U: \mathbb{V} \rightarrow \mathbb{V}$ be a linear operator and $g U=\{(x, y) \in \mathbb{V} \times \mathbb{V} \mid y=U x\}$ be the graph of $U$. A lagrangian subspace of $\vee \times \mathbb{V}$ is an $n$-dimensional subspace on which $\omega$ vanishes. It can be checked directly that $g U$ is lagrangian if and only if $U$ is self-adjoint. Moreover for a self-adjoint $U, g U \subset \mathbb{C}$ if and only if $U \geq 0$.

If $S$ is symplectic and $Q$-monotone and $U \geq 0$ then $S g U$ is a lagrangian subspace contained in $\mathbb{C}$. Moreover $S g U$ is the graph of an operator which we denote by $S U$ 
( $g S U=\dot{S} S g U$ ). Indeed we have to check that $S g U$ does not contain $v=(0, y), y \neq 0$. If it does then $S^{-1} v=(x, U x)$ and $\langle x, U x\rangle=0$ so that $U x=0$. Further $0=A x+$ $B U x=A x$ which contradicts the invertibility of $A$.

Clearly if $U>0$ then $S U>0$. We have

$$
S U=(C+D U)(A+B U)^{-1} .
$$

For a self adjoint $U>0$ the restriction of $Q$ to $g U$ and also $S g U$ is a positive definite quadratic form i.e. a scalar product. By $\alpha(U, S)$ we denote the coefficient of volume expansion under the action of $S$ from the subspace $g U$ onto $S g U$, where both subspaces are equipped with scalar products obtained by restricting $Q$.

LEMMA 2.

$$
\alpha(U, S)=\sqrt{\frac{\operatorname{det}(A+B U) \operatorname{det}(C+D U)}{\operatorname{det} U}} .
$$

Proof. We introduce linear coordinates in $g U$ and $S g U$ by the projection $\pi_{1}: \mathbb{V} \times \mathbb{V} \rightarrow$ $\mathbb{V}$ on the first component. The restriction of $Q$ to these subspaces is transformed by $\pi_{1}$ into the quadratic forms $\langle U \cdot, \cdot\rangle$ and $\langle S U \cdot, \cdot\rangle$ respectively. The action of $S$ in these coordinates is given by the formula

$$
\mathbb{V} \ni x \mapsto A x+B U x \in \mathbb{V} \text {. }
$$

Hence, by (3),

$$
\begin{aligned}
\alpha(U, S) & =\sqrt{\operatorname{det} S U}|\operatorname{det}(A+B U)| / \sqrt{\operatorname{det} U} \\
& =\frac{\sqrt{\operatorname{det}(A+B U) \operatorname{det}(C+D U)}}{\sqrt{\operatorname{det} U}} .
\end{aligned}
$$

THEOREM 3. Let $S$ be a symplectic Q-monotone linear operator. Then

$$
\alpha(U, S) \geq \rho(S)
$$

for every $U>0$.

Proof. Let

$$
S=\left(\begin{array}{cc}
A & B \\
C & D
\end{array}\right) \text { and } S_{1}=\left(\begin{array}{cc}
A^{-1} & 0 \\
0 & A^{*}
\end{array}\right)\left(\begin{array}{cc}
A & B \\
C & D
\end{array}\right)=\left(\begin{array}{cc}
I & R \\
P & I+P R
\end{array}\right)
$$

where $R=A^{-1} B \geq 0$ and $P=A^{*} C \geq 0$. We have $\alpha(U, S)=\alpha\left(U, S_{1}\right)$ because the symplectic map

$$
\left(\begin{array}{cc}
A^{-1} & 0 \\
0 & A^{*}
\end{array}\right)
$$

preserves $Q$. Also $C^{*} B=P R$ so that

$$
\rho(S)=\rho\left(S_{1}\right) .
$$

Hence it is enough to establish (4) only for

$$
S=\left(\begin{array}{cc}
I & R \\
P & I+P R
\end{array}\right)
$$

$P \geq 0, R \geq 0 . \alpha(U, S)$ depends continuously on $S$ and so does $\rho(S)$, This allows further restriction to the case $P>0$ and $R>0$. 
Now we can simplify $S$ by the following transformation

$$
S_{1}=\left(\begin{array}{cc}
(\sqrt{R})^{-1} & 0 \\
0 & \sqrt{R}
\end{array}\right)\left(\begin{array}{cc}
I & R \\
P & I+P R
\end{array}\right)\left(\begin{array}{cc}
\sqrt{R} & 0 \\
0 & \sqrt{R^{-1}}
\end{array}\right)=\left(\begin{array}{cc}
I & I \\
T & I+T
\end{array}\right)
$$

where $T=\sqrt{R} P \sqrt{R}$ has the same eigenvalues as $P R=(\sqrt{R})^{-1} T \sqrt{R}$. Hence $\rho\left(S_{1}\right)=$ $\rho(S)$. Clearly $\alpha(U, S)=\alpha\left(U_{1}, S_{1}\right)$ where $U_{1}=\sqrt{R} U \sqrt{R}$. So finally it is enough to establish (4) only for

$$
S=\left(\begin{array}{cc}
I & I \\
T & I+T
\end{array}\right)
$$

$T>0$. For such $S$

$$
\alpha(U, S)=\frac{\sqrt{\operatorname{det}(T+(I+T) U) \operatorname{det}(I+U)}}{\sqrt{\operatorname{det} U}}=\frac{\sqrt{\operatorname{det}\left(T+U(I+U)^{-1}\right)} \operatorname{det}(I+U)}{\sqrt{\operatorname{det} U}} .
$$

In general if $A$ and $B$ are self-adjoint positive definite operators then

$$
\operatorname{det}(A+B)>\operatorname{det} A \text {. }
$$

Hence

and further

$$
\operatorname{det}\left(T+U(I+U)^{-1}\right)>\operatorname{det} U / \operatorname{det}(I+U)
$$

$$
\alpha(U, S)>\sqrt{\operatorname{det}(I+U)}>1 .
$$

Similarly

$$
\alpha(U, S)>\sqrt{\operatorname{det} T} \operatorname{det}\left((\sqrt{U})^{-1}+\sqrt{U}\right) .
$$

The last inequality shows that $\alpha(U, S) \rightarrow \infty$ when one of the eigenvalues of $U$ goes to 0 or $+\infty$. We conclude that $\alpha(U, S)$ has an absolute minimum at some $U_{0}>0$ and $\alpha\left(U_{0}, S\right)>1$. To find this minimum we will differentiate $\alpha(U, S)$ with respect to $U$. It turns out that $\alpha(U, S)$ has a unique critical point which hence must be the minimum.

LEMMA 3. Let $f(U)=\ln \alpha(U, S)$ for $U>0$ then

$$
D f(Z)=\frac{1}{2}\left(\operatorname{tr} Z\left[(I+T)^{-1} T+U\right]^{-1}+\operatorname{tr} Z(I+U)^{-1}-\operatorname{tr} Z U^{-1}\right)
$$

where the derivative is taken in the manifold of positive self-adjoint operators $U$ so that $Z$ is an arbitrary self-adjoint operator.

Proof. If $g(U)=\ln$ det $U$ then

$$
D g(Z)=\operatorname{tr} Z U^{-1} \text {. }
$$

Using the chain rule we get our formula by straightforward computation.

It follows from lemma 3 that $\alpha(U, S)$ has a critical point at $U$ if

$$
\left((I+T)^{-1} T+U\right)^{-1}+(I+U)^{-1}-U^{-1}=0 .
$$

Transforming the equation we get

$$
U^{2}=(I+T)^{-1} T^{2}
$$

There is a unique positive definite $U_{0}$ which satisfies this equation. Moreover,

$$
\alpha\left(U_{0}, S\right)=\sqrt{\operatorname{det}\left((I+T) U_{0}+T U_{0}^{-1}+T+(I+T)\right)}
$$


and

so that

$$
(I+T) U_{0}=T U_{0}^{-1}=\sqrt{T} \sqrt{I+T}
$$

$$
\alpha\left(U_{0}, S\right)=\operatorname{det}(\sqrt{I+T}+\sqrt{T})=\rho(S)
$$

\section{Lyapunov exponents}

Let $T: X \rightarrow X$ be a measurable transformation of the measurable space $X$ preserving the probabilistic measure $\mu$ and let $S: X \rightarrow \operatorname{Sp}(\mathbb{V})$ be a measurable map with values in symplectic operators such that

$$
\int_{X} \ln ^{+}\|S(x)\| d \mu(x)<+\infty \quad \text { where } \ln ^{+} a=\max (a, 0) .
$$

By the Multiplicative Ergodic Theorem of Oseledec (see [Os], [Rue]), we have that for $\mu$-almost all $x \in X$ the following limit exists

$$
\lim _{k \rightarrow+\infty}\left(S^{k^{*}}(x) S^{k}(x)\right)^{1 / 2 k}=\Lambda(x)
$$

where

$$
S^{k}(x)=S\left(T^{k-1} x\right) \cdot \ldots \cdot S(x) .
$$

The logarithms of eigenvalues of $\Lambda$ are called Lyapunov exponents.

In our symplectic case $\Lambda$ is also symplectic. Indeed the symplecticity of $S^{k}$ means that

$$
S^{k^{*}} J S^{k}=J
$$

Taking into account that $J^{-1}=-J$ we get

$$
J^{-1} S^{k} J=\left(S^{k^{*}}\right)^{-1} \text { and } J^{-1} S^{k^{*}} J=\left(S^{k}\right)^{-1} \text {. }
$$

It follows that

$$
J^{-1} S^{k^{*}} S^{k} J=\left(S^{k^{*}} S^{k}\right)^{-1} \text { and } J^{-1}\left(S^{k^{*}} S^{k}\right)^{1 / 2 k} J=\left(S^{k^{*}} S^{k}\right)^{-1 / 2 k}
$$

Taking the limit we obtain

$$
J^{-1} \Lambda J=\Lambda^{-1}
$$

which together with $\Lambda^{*}=\Lambda$ means that $\Lambda$ is symplectic.

We obtain that the non-zero Lyapunov exponents appear in pairs $\lambda,-\lambda$ i.e. the eigenvalues of $\Lambda$ are

$$
e^{-\lambda_{s}}<\cdots<e^{-\lambda_{1}}<1<e^{\lambda_{1}}<e^{\lambda_{2}}<\cdots<e^{\lambda_{s}}
$$

where 1 may or may not be one of the eigenvalues. Moreover the multiplicities of $e^{\lambda_{i}}$ and $e^{-\lambda_{i}}$ are equal and we denote them by $d_{i}$. Indeed let $E_{i}, i=0, \pm 1, \ldots, \pm s$ be the eigenspace of the corresponding eigenvalue. If 1 is not an eigenvalue we put $E_{0}=\{0\}$. We have $J E_{i}=E_{-i}$.

Let $E^{s}=E_{-1} \oplus \cdots \oplus E_{-s}$.

LEMMA 4. $E^{s} \oplus E_{0}$ is the skew orthogonal complement of $E^{s}$.

Proof. $E_{i}, i=0, \pm 1, \ldots, \pm s$ are mutually orthogonal and $J E_{i}=E_{-i}$. It follows that $E_{i}$ is skew orthogonal to $E_{j}$ if $j \neq-i$. Consequently $E^{s} \oplus E_{0}$ is skew orthogonal to $E^{s}$ and since they have complementary dimensions one is the skew orthogonal complement of the other. 
By the Multiplicative Ergodic Theorem, for $\mu$-almost all $x \in X$ if a subspace $E \subset \mathbb{V} \times \mathbb{V}$ is such that $E^{s} \subset E \subset E^{s} \oplus E_{0}$ then

$$
\lim _{k \rightarrow+\infty} \frac{1}{k} \ln \left|\operatorname{det}\left(\left.S^{k}\right|_{E}\right)\right|=-\left(\lambda_{1} d_{1}+\cdots+\lambda_{s} d_{s}\right)
$$

and if $E$ is such that $E^{s} \cap E=\{0\}$ and $E^{s} \oplus E_{0} \oplus E=\mathbb{V} \times \mathbb{V}$ then

$$
\lim _{k \rightarrow+\infty} \frac{1}{k} \ln \left|\operatorname{det}\left(\left.S^{k}\right|_{E}\right)\right|=\lambda_{1} d_{1}+\cdots+\lambda_{s} d_{s} \text {. }
$$

In other words the exponential rate of volume growth on the subspace $E$ under the action of $S^{k}$ is equal to the sum of respectively negative and positive Lyapunov exponents.

LEMMA 5. For a lagrangian subspace $E$ of $\mathbb{V} \times \mathbb{V}$

(a) $E^{s} \subset E$ if and only if $E \subset E^{s} \oplus E_{0}$;

(b) $E^{s} \cap E=\{0\}$ if and only if $E^{s} \oplus E_{0} \oplus E=\mathbb{V} \times \mathbb{V}$.

Proof. (a) $E^{s} \subset E$ if and only if the skew orthogonal complement of $E^{s}$, which by lemma 4 , is equal to $E^{s} \oplus E_{0}$, contains the skew orthogonal complement of $E$, which by the definition of a lagrangian subspace is equal to $E$.

(b) $E^{s} \cap E$ is the skew orthogonal complement of $E^{s} \oplus E_{0} \oplus E$.

LemMA 6. There is a lagrangian subspace containing $E^{s}$ and the set of lagrangian subspaces $E$ such that $E^{s} \cap E=\{0\}$ is open and dense in the lagrangian grassmanian.

Proof. If $E_{0}=\{0\}$ then, by lemma $4, E^{s}$ is lagrangian. If $E_{0} \neq\{0\}$ then it is a symplectic subspace. Indeed $J E_{0}=E_{0}$ so that the restriction of $\omega$ to $E_{0}$ is nondegenerate. Let $L \subset E_{0}$ be a lagrangian subspace of the symplectic space $E_{0}$ then $E^{s} \oplus L$ is a lagrangian subpace in $V \times V$ (actually every lagrangian subspace containing $E^{s}$ can be obtained in this way).

The second claim in the lemma follows from the first and from the general fact that the set of lagrangian subspaces transversal to a particular lagrangian subspace is open and dense in the lagrangian grassmanian.

It follows from the above discussion that if we are interested in the sum of the positive Lyapunov exponents then it is natural to consider volume growth on lagrangian subspaces only. Note in particular that if there are no zero exponents then $E^{s}$ is lagrangian.

We are now able to formulate and prove a fundamental estimate for the sum of positive Lyapunov exponents in the case of $Q$-monotone symplectic operators. It follows easily from the estimate in theorem 3.

THEOREM 4. If $S: X \rightarrow \mathrm{Sp}(\mathbb{V})$ has values in Q-monotone operators then

$$
\int_{X}\left(\lambda_{1} d_{1}+\cdots+\lambda_{s} d_{s}\right) d \mu \geq \int_{X} \ln \rho(S(x)) d \mu .
$$

Remark. This theorem can be applied to a single $Q$-monotone symplectic matrix

$$
S=\left(\begin{array}{ll}
A & B \\
C & D
\end{array}\right)
$$


It yields the inequality

$$
\left|\nu_{1}\right|\left|\nu_{2}\right| \cdots\left|\nu_{n}\right| \geq \prod_{i=1}^{n}\left(\sqrt{1+u_{i}}+\sqrt{u_{i}}\right)
$$

where $\nu_{1}, \ldots, \nu_{n}$ are the eigenvalues of $S$ outside the unit circle and $u_{1}, \ldots, u_{n}$ are the eigenvalues of $C^{*} B$.

Proof. By Lemma 6 there are self-adjoint positive definite operators $U: \mathbb{V} \rightarrow \mathbb{V}$ such that $E^{s} \cap g U=\{0\}$ (it is not difficult to see that it holds actually for every $U>0$ ).

$\langle x, x\rangle+\langle y, y\rangle \geq\langle x, y\rangle$ for every $x, y \in V$ so that the standard scalar product on any subspace $g U, U>0$, is bigger than the restriction of $Q$. It follows that

$$
\lambda_{1} d_{1}+\cdots+\lambda_{s} d_{s}=\lim _{k \rightarrow+\infty} \frac{1}{k} \ln \left|\operatorname{det}\left(\left.S^{k}(x)\right|_{g U}\right)\right| \geq \limsup _{k \rightarrow+\infty} \frac{1}{k} \ln \alpha\left(U, S^{k}(x)\right) .
$$

By theorem 3,

$$
\begin{aligned}
\alpha\left(U, S^{k}(x)\right) & =\alpha(U, S(x)) \alpha(S(x) U, S(T x)) \cdots \alpha\left(S^{k-1}(x) U, S\left(T^{k-1} x\right)\right) \\
& \geq \rho\left(S(x) \rho(S(T x)) \cdots \rho\left(S\left(T^{k-1} x\right)\right)\right) .
\end{aligned}
$$

Herce

$$
\lambda_{1} d_{1}+\cdots+\lambda_{s} d_{s} \geq \limsup _{k \rightarrow \infty} \frac{1}{k} \sum_{i=0}^{k-1} \ln \rho\left(S\left(T^{i} x\right)\right)
$$

Since a priori we do not know if $\ln \rho(S(x))$ is integrable we cannot use the Birkhoff Ergodic Theorem. Nevertheless we obtain our estimate from the following general lemma.

LeMmA 7. Let $T: X \rightarrow X$ preserve a probabilistic measure $\mu$ on $X$ and f be a measurable function on $X, f \geq 0$. If for some measurable function $g$ and almost every $x \in X$

$$
\lim _{n \rightarrow+\infty} \frac{1}{n} \sum_{i=1}^{n-1} f\left(T^{i} x\right) \leq g(x)
$$

then

$$
\int_{X} f(x) d \mu \leq \int_{X} g(x) d \mu .
$$

Proof. Let

$$
f_{N}(x)= \begin{cases}f(x) & \text { if } f(x) \leq N \\ N & \text { if } f(x) \geq N .\end{cases}
$$

We have $0 \leq f_{N} \leq f$ and $f_{N}$ is integrable. By the Birkhoff Ergodic Theorem we obtain

$$
\lim _{n \rightarrow+\infty} \frac{1}{n} \sum_{i=1}^{n-1} f_{N}\left(T^{i} x\right)=f_{N}^{*}(x)
$$

and

$$
\int_{X} f_{N}^{*}(x) d \mu=\int_{X} f_{N}(x) d \mu .
$$

But $f_{N}^{*}(x) \leq g(x)$ so that

$$
\int_{X} f_{N}(x) d \mu \leq \int_{X} g(x) d \mu .
$$




\section{Gas of hard spheres}

We will consider the system of $N$ identical $d$-dimensional balls in the $d$-dimensional torus $\mathbb{T}^{d}=\mathbb{R}^{d} / \mathbb{Z}^{d}$. The radius of a ball $r$ cannot exceed a certain $r_{0}=r_{0}(N)$. We assume that the balls move uniformly and collide elastically.

Let $q_{i} \in \mathbb{R}^{d}, q_{i} \bmod 1$ be the position of the center of the $i$-th ball and $v_{i} \in \mathbb{R}^{d}$ its velocity, $i=1, \ldots, N$. The configuration space $Q$ of our system is

$$
Q=\left\{\left(q_{1}, \ldots, q_{N}\right) \bmod 1|| q_{i}-q_{j}+k \mid \geq 2 r \text { for every } k \in \mathbb{Z}^{d}\right\}
$$

The boundary of $Q$ is piecewise smooth and the smooth pieces are isometric to pieces of the cylinder $\left\{\left(z_{1}, \ldots, z_{N}\right) \in\left(\mathbb{R}^{d}\right)^{N}|| z_{1} \mid=\sqrt{2} r\right\}$. In particular the boundary of $Q$ has $d-1$ principal curvatures equal to $1 / \sqrt{2} r$ and the rest are zero.

We fix the total energy of the system $N E=\frac{1}{2} \sum_{i=1}^{N} v_{i}^{2}$. The phase space is the bundle of spheres of radius $\sqrt{2 N E}$ over $Q$ which we denote by $F$. The dynamics of our system is described by a flow $\Phi^{t}$ in $F$ which preserves the Liouville measure $\nu$ (the microcanonical probability distribution).

It turns out that the elastic collisions of balls lead to billiard dynamics i.e. $\Phi^{\prime}$ describes the dynamics of a mass point moving uniformly with speed $\sqrt{2 N E}$ in $Q$ and reflecting elastically from the boundary of $Q$ (at reflection the tangential component of the velocity is preserved and the normal component is reversed).

We will estimate the measure theoretic entropy $h\left(\Phi^{1}\right)$ of $\Phi^{1}$ from below using Theorem 4.

To that end let us consider the standard section map $T: \Sigma \rightarrow \Sigma$ where $\Sigma$ consists of vectors (of length $\sqrt{2 N E}$ ) attached at the boundary of $Q$ and pointing inwards. $T$ is the first return map for the billiard flow $\Phi^{t}$. By $\tau: \Sigma \rightarrow \mathbb{R}^{+}$we denote the time of the first return i.e. the time to the next collision. $T$ preserves the natural smooth measure $\mu$ (we do not describe it explicitly because we will use the measure $\nu$ in the final formula). The measure theoretic entropies of $\Phi^{1}$ and $T$ are connected by the Abramov formula

$$
h\left(\Phi^{1}\right)=h(T) / \int_{\Sigma} \tau d \mu .
$$

Now our goal is to describe $D T$. We begin with introducing convenient coordinates in the tangent spaces of $\Sigma$ at $p \in \Sigma$ and $T p \in \Sigma$. Let $\pi: \Sigma \rightarrow \partial Q$ be the natural projection.

By $\mathbb{L}_{p}$ we denote the tangent space of $\partial Q$ at $\pi(p)$ and by $\mathbb{V}_{p}$ the linear subspace orthogonal to the vector $p$. The tangent space of $\Sigma$ at $p$ can be naturally identified with $\mathbb{L}_{p} \times \mathbb{V}_{p}$. Let $P: \mathbb{V}_{p} \rightarrow \mathbb{L}_{p}$ be the projection along $p$. We identify $\mathbb{L}_{p} \times \mathbb{V}_{p}$ with $\mathbb{V}_{p} \times \mathbb{V}_{p}$ via the linear operator $P \times I: \mathbb{V}_{p} \times \mathbb{V}_{p} \rightarrow \mathbb{L}_{p} \times \mathbb{V}_{p}$ where $I$ is the identity operator on $\mathbb{V}_{p}$. Further we identify $\mathbb{V}_{p}$ and $\mathbb{V}_{T_{p}}$ by translating $\mathbb{V}_{p}$ parallely to $\pi(T p)$ and reflecting it in the tangent plane $L_{T p}$.

After these identifications $D_{p} T$ becomes an operator on $\mathbb{V}_{p} \times \mathbb{V}_{p}$. The computation of $D_{p} T$ is performed in Appendix B and the result is the following block operator

$$
\left|\begin{array}{cc}
I & \tau \sqrt{2 N E I} \\
\frac{1}{\sqrt{2 N E}} R & I+\tau R
\end{array}\right|
$$

where $R$ is a self-adjoint operator with $d-1$ nonzero eigenvalues: $(r \cos \psi)^{-1}\left|V_{\text {rel }}\right|$ 
of multiplicity 1 and $r^{-1} \cos \psi\left|V_{\text {rel }}\right|$ of multiplicity $d-2 ; V_{\text {rel }}=V_{i}-V_{j}$ is the relative velocity of the colliding $i$ th and $j$ th spheres and $\psi$ is the angle between $V_{\text {rel }}$ and the line through the centers of the colliding spheres at the moment of collision. In particular $D_{p} T$ is a $Q$-monotone symplectic operator with respect to the standard symplectic structure in $\mathbb{V}_{p} \times \mathbb{V}_{p}$.

$T$ has singularities but it follows from [Led-Str] (see also [Kat-Str]) that at least the estimate of the entropy from below in the Pesin formula holds for $T$. Hence using theorem 4 we obtain

$$
h(T) \geq \int_{\Sigma}\left(\sinh ^{-1} \sqrt{(r \cos \psi)^{-1} \tau\left|V_{\mathrm{rel}}\right|}+(d-2) \sinh ^{-1} \sqrt{r^{-1} \cos \psi \tau\left|V_{r e l}\right|}\right) d \mu .
$$

We keep only the first term in the integral and replace the integration with respect to $d \mu$ by the integration with respect to the microcanonical probability distribution $d \nu=d t d \mu / \int_{\Sigma} \tau d \mu$ :

$$
\begin{aligned}
h\left(\Phi^{1}\right) & \geq \frac{\int_{\Sigma} \sinh ^{-1} \sqrt{(r \cos \omega)^{-1} \tau \mid V_{\text {rel }}} d \mu}{\int_{\Sigma} \tau d \mu} \\
& =\int_{F} \tau^{-1} \sinh ^{-1} \sqrt{(\mathrm{r} \cos \psi)^{-1} \tau\left|V_{\text {rel }}\right|} d \nu .
\end{aligned}
$$

In the last integral we treat $\tau, V_{\text {rel }}$ and $\psi$ as functions on the phase space $F$ in the following sense:

$\tau$ is the time between the nearest collision in the past and the nearest collision in the future, $V_{\text {rel }}$ and $\psi$ correspond to the nearest future collision.

We cannot show rigorously that the integral (5) goes to $+\infty$ as the radius of the spheres $r$ approaches the maximal radius $r_{0}(N)$. Nevertheless we feel that it is so beyond reasonable doubt. Here is one way to see it. The integral (5) is bigger than

$$
\int_{F} \tau^{-1} \sinh ^{-1} \sqrt{r^{-1} \tau\left|V_{\text {rel }}\right|} d \nu
$$

Let $f(a)=a^{-1} \sinh ^{-1} \sqrt{a}$ for $a>0$. We have $f(a)=a^{-\frac{1}{2}}+O(1)$ for small $a$. It can be checked that $f$ is a concave function i.e. $f^{\prime \prime}>0$. To estimate from below the integral (6) we use the Jensen inequality for the function $f$. We get

$$
\int_{F} f\left(r^{-1} \tau\left|V_{\text {rel }}\right|\right) r^{-1}\left|V_{\text {rel }}\right| d \nu \geq r^{-1} \int_{F}\left|V_{\text {rel }}\right| d \nu f\left(r^{-1} \int_{F} \tau\left|V_{\text {reel }}\right|^{2} d \nu / \int_{F}\left|V_{\text {rell }}\right| d \nu\right) \text {. }
$$

Clearly $\int_{F}\left|V_{\text {rell }}\right| d \nu$ is bounded away from zero uniformly in $r<r_{0}, \tau\left|V_{\text {rel }}\right|$ describes roughly speaking how far apart two colliding spheres could be at the moment of the nearest past collision (of some may be different spheres). It is clear that when $r$ is very close to $r_{0}$ most of the spheres almost cannot move, so that $\tau\left|V_{\text {rel }}\right|$ is very small with large probability. It should be also very small when averaged with respect to $\left|V_{\text {rel }}\right| d \nu$ and so (7) is very large when $r$ is close to $r_{0}$.

We believe that for certain values of $N$ (when the spheres are well packed into the torus) the uniform smallness of $\tau\left|V_{\text {rel }}\right|$ can be established making the above argument rigorous. When the number of spheres $N$ is such that they can fill the 
torus only with considerable gaps then $\tau\left|V_{\text {rel }}\right|$ is not uniformly small for $r$ close to $r_{0}$ and then the rigorous estimate of (7) seems to be a cumbersome task.

Another nonrigorous way to see that (7) goes to $+\infty$ when $r \rightarrow r_{0}(N)$ is the iollowing. For large $N$ the time $\tau$ between consecutive collisions and $\left|V_{\text {rel }}\right|$ are nearly independent so that

$$
\begin{gathered}
\int_{F} \tau\left|V_{\mathrm{rel}}\right|^{2} d \nu \approx \int_{F} \tau d \nu \int_{F}\left|V_{\mathrm{rel}}\right|^{2} d \nu \\
\int_{F}\left|V_{\mathrm{rei}}\right|^{2} d \nu / \int_{F}\left|V_{\mathrm{rel}}\right| d \nu \geq \int_{F} \min _{i, j}\left|v_{i}-v_{j}\right|^{2} d \nu / \int_{F} \max _{i, j}\left|v_{i}-v_{j}\right| d \nu
\end{gathered}
$$

The last expression does not depend on $r$ (but it does depend on $N$ ). Hence (7) is large if only the average time between collisions $\int_{F} \tau d \nu$ is small.

\section{Appendix A}

We are going to compute three integrals

$$
a=\int_{\Sigma_{0}} \frac{2}{\sin \phi_{1}} d \mu, \quad b=\int_{\Sigma_{0}} \tau d \mu \text { and } c=\int_{\Sigma_{0}} \sqrt{\frac{2 \tau}{\sin \phi_{1}}} d \mu .
$$

(we listed them in the order of increasing difficulty).

Since $T$ preserves the measure $\mu$

$$
a=\int_{\Sigma_{0}} \frac{2}{\sin \phi} d \mu=\int_{\Sigma_{0}} \frac{2}{\sin \phi} \sin \phi d s d \phi / \int_{\Sigma_{0}} \sin \phi d s d \phi=8 \pi / 8=\pi .
$$

To compute $b$ and $c$ let us consider the probabilistic measure $\nu$ in $S_{1} Q_{0}$ preserved by the billiard flow. In $S_{1} Q_{0}$ we introduce two systems of coordinates. One is $(x, y, \psi)$, where $(x, y) \in Q_{0}$ and $\psi$ is the angle with the $x$-axis. The other one is $(s, \phi, t)$, where $(s, \phi) \in \Sigma_{0}$ and $t, 0 \leq t \leq \tau$, is the time parameter along the orbit starting at $(s, \phi) \in \Sigma_{0}$. In these coordinates we have

$$
d \nu=(2 \pi)^{-1} d x d y d \psi=(2 \pi)^{-1} \sin \phi d s d \phi d t
$$

Hence

$$
1=(2 \pi)^{-1} \int_{S_{1} Q_{1}} \sin \phi d s d \phi d t=(2 \pi)^{-1} \int_{\Sigma_{0}} \tau \sin \phi d s d \phi=4 \pi^{-1} \int_{\Sigma_{0}} \tau d \mu
$$

and $b=\pi / 4$. Finally

$$
c=\int_{S_{1} Q_{0}} \sqrt{\frac{2 \tau}{\sin \phi_{1}}} \frac{1}{\tau} d t d \mu=\frac{\sqrt{2} \pi}{4} \int_{S_{1} Q_{0}}\left(\tau \sin \phi_{1}\right)^{-\frac{1}{2}} d \nu
$$

where we treat $\tau$ and $\phi_{1}$ as functions on $S_{1} Q_{0}$, constant on the billiard orbit between consecutive reflections.

Using the symmetries of the square $Q_{0}$ we obtain

$$
c=\frac{\sqrt{2}}{8} \int_{0}^{2 \pi}\left(\iint_{Q_{0}}\left(\tau \sin \phi_{1}\right)^{-\frac{1}{2}} d x d y\right) d \psi=\sqrt{2} \int_{0}^{\pi / 4}\left(\iint_{Q_{0}}\left(\tau \sin \phi_{1}\right)^{-\frac{1}{2}} d x d y\right) d \psi
$$

For a fixed direction $\psi$ we rotate the coordinate system in $Q_{0}$ so that $x$ becomes the coordinate along the direction $\psi$ and $y$ is the coordinate in the perpendicular direction. Then $\tau$ and $\sin \phi_{1}$ become functions of $y$ alone and for a fixed $y, x$ 
changes in an interval of length $\tau$. We get

$$
\iint_{Q_{0}}\left(\tau \sin \phi_{1}\right)^{-\frac{1}{2}} d x d y=\int \sqrt{\frac{\tau}{\sin \phi_{1}}} d y
$$

In the last integral $\tau$ is a piecewise linear function of $y$ and $\sin \phi_{1}$ is piecewise constant. By elementary geometry (see figure 2 ) we obtain

$$
\begin{aligned}
\int \sqrt{\frac{\tau}{\sin \phi_{1}}} d y= & \int_{-\sin \psi}^{0} \sqrt{\frac{y+\sin \psi}{\sin \psi \cos ^{2} \psi}} d y+\int_{0}^{\cos \psi-\sin \psi} \frac{1}{\cos \psi} d y \\
& +\int_{\cos \psi-\sin \psi}^{\cos \psi} \sqrt{\frac{\cos \psi-y}{\sin ^{2} \psi \cos \psi}} d y=1-\frac{1}{3} \tan \psi+\frac{2}{3} \sqrt{\tan \psi}
\end{aligned}
$$

Finally by elementary integration

$$
c=\sqrt{2} \int_{0}^{\pi / 4}\left(1-\frac{1}{3} \tan \psi+\frac{2}{3} \sqrt{\tan \psi}\right) d \psi=\left(\frac{\sqrt{2}}{4}+\frac{1}{3}\right) \pi-\frac{\sqrt{2}}{6} \ln 2-\frac{1}{3} \ln (3+\sqrt{2}) .
$$

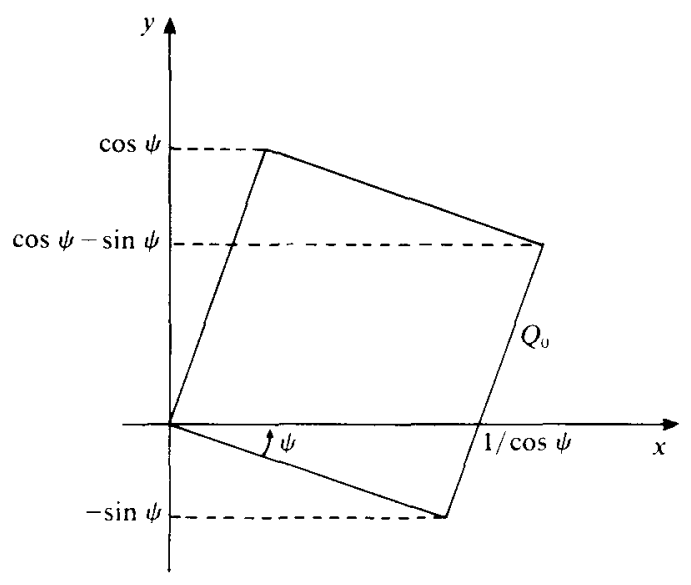

FIGURE 2

\section{Appendix $B$}

We are going to compute the derivative of the standard section map $T: \Sigma \rightarrow \Sigma$ of the billiard flow. The results of this computation are essentially formulated in the papers [Sin 2], [Sin 3], [Cher], [Cher-Sin] but the detailed derivation is not supplied there.

$D_{p} T$ is the composition of two linear maps: the first one corresponds to the free transfer up to the point of reflection, the other one describes the reflection itself.

The free transfer is described by the usual Jacobi equation for the orthogonal component of the Jacobi field. In our case the metric is flat so that the Jacobi equation is especially simple

$$
\begin{aligned}
& \dot{x}=y, \\
& \dot{y}=0,
\end{aligned}
$$

where $(x, y) \in \mathbb{V}_{p} \times \mathbb{V}_{p}$. 
If the distance between consecutive collisions is $s$ then the linear map is

$$
(x, y) \mapsto(x+s y, y)
$$

or in the block form

$$
\left|\begin{array}{cc}
I & s I \\
0 & I
\end{array}\right|
$$

Hence this linear map is symplectic with respect to the standard symplectic structure in $\mathbb{V}_{p} \times \mathbb{V}_{p}\left(\mathbb{V}_{p}\right.$ is equipped with the standard scalar product). The action of this map on the lagrangian subspaces $y=U x, U^{*}=U$ is given by $U \rightarrow U(I+s U)^{-1}$.

We will not study the action of the linear map corresponding to the reflection on an individual vector but will rather find out how it acts on lagrangian subspaces of $\mathbb{V}_{p} \times \mathbb{V}_{p}$. This action can be described in the following way. At the moment of reflection we have two rays: one incoming with the direction $\xi_{0}^{-}$and the reflected ray with the direction $\xi_{0}^{+}$. Let us consider a local hypersurface $S_{-}$passing through the point of reflection and orthogonal to $\xi_{0}^{-}$. The family of rays orthogonal to $S_{-}$ is infinitesimally described by the operator $U_{-}$of the second fundamental form of $S_{-}$at the point of reflection. The family of unit vectors orthogonal to $S_{-}$is a submanifold in our phase space and its tangent subspace is the graph of $U_{-}$in $\mathbb{V}_{p} \times \mathbb{V}_{p}$, which is a lagrangian subspace since $U_{-}$is self-adjoint. It is well known in the geometric optics that if we reflect all the rays of our family in the boundary of $Q$ we will obtain the family of rays orthogonal to a certain local hypersurface $S_{+}$passing through the point of reflection (and orthogonal to $\xi_{0}^{+}$). If we use $w \in \mathbb{R}_{T p}$ as local coordinates on $\partial Q$ then we can write the family of incoming unit vectors as

$$
\xi^{-}=\xi_{0}^{-}+U_{-} P_{1}^{-1} w+O\left(|w|^{2}\right)
$$

and the family of reflected vectors as

$$
\xi^{+}=\xi_{0}^{+}+U_{+} P_{2}^{-1} w+O\left(|w|^{2}\right),
$$

where $U_{+}$is the operator of the second fundamental form of $S_{+}$(at the point of reflection), $P_{1}: \mathbb{V}_{p} \rightarrow \mathbb{L}_{T p}$ is the projection along $\xi_{0}^{-}$and $P_{2}: \mathbb{V}_{T_{p}} \rightarrow \mathbb{L}_{T p}$ is the projection along $\xi_{0}^{+}$. Note that $P_{2}^{-1} P_{1}: \mathbb{V}_{p} \rightarrow \mathbb{V}_{T p}$ is the reflection in $\mathbb{L}_{T p}$ which identifies $\mathbb{V}_{p}$ and $\mathbb{V}_{T p}$. The derivative $D T$ takes the graph of $U_{-}$into the graph of $U_{+}$. To find the connection between $U_{-}$and $U_{+}$we will use the fact that $\xi^{+}$is the reflection of $\xi^{-}$ in the respective tangent space of $\partial Q$. Using again $w \in \mathbb{L}_{T p}$ as a local coordinate on $\partial Q$ we can write the inside unit normal vector to $\partial Q$ as

$$
n=n_{0}+K w+O\left(|w|^{2}\right),
$$

where $K$ is the operator of the second fundamental form of $\partial Q$ at the point of reflection, $n_{0}$ is the inside unit normal vector of the point of reflection. (In our special case the operator $K$ is positive semidefinite.)

We have

$$
\xi^{+}=\xi^{-}-2\left\langle\xi^{-}, n\right\rangle n
$$

Expanding both sides up to the linear terms in $w$ we get

$$
\begin{aligned}
\xi_{0}^{+}+U_{+} P_{2}^{-1} w+\cdots= & \xi_{0}^{-}+U_{-} P_{1}^{-1} w-2\left\langle\xi_{0}^{-}, n_{0}\right\rangle n_{0} \\
& -2\left\langle\xi_{0}^{-}, n_{0}\right\rangle K w-2\left\langle\xi_{0}^{-}, K w\right\rangle n_{0}-2\left\langle U_{-} P_{1}^{-1} w, n_{0}\right\rangle n_{0}+\cdots
\end{aligned}
$$


Using the fact that

$$
U_{-} P_{1}^{-1} w-2\left\langle U_{-} P_{1}^{-1} w, n_{0}\right\rangle n_{0}=P_{2}^{-1} P_{1} U_{-} P_{1}^{-1} w
$$

we obtain

$$
U_{+}=P_{2}^{-1} P_{1} U_{-} P_{1}^{-1} P_{2}+2\left\langle\xi_{0}^{+}, n_{0}\right\rangle K P_{2}-2\left\langle\xi_{0}^{+}, K P_{2} \cdot\right\rangle n_{0} .
$$

We need yet to identify the operator

$$
w \rightarrow 2\left\langle\xi_{0}^{+}, n_{0}\right\rangle K w-2\left\langle\xi_{0}^{+}, K w\right\rangle n_{0} .
$$

The operator

$$
\mathbb{L}_{T p} \ni w \rightarrow w-\frac{\left\langle\xi_{0}^{+}, w\right\rangle}{\left\langle\xi_{0}^{+}, n_{0}\right\rangle} n_{0} \in \mathbb{V}_{T_{p}}
$$

is the projection along $n_{0}$ which is also equal to $P_{2}^{*}: \mathbb{L}_{T_{p}} \rightarrow \mathbb{V}_{T p}$. Hence finally

$$
U_{+}=P_{2}^{-1} P_{1} U_{-} P_{1}^{-1} P_{2}+2\left\langle\xi_{0}^{+}, n_{0}\right\rangle P_{2}^{*} K P_{2} \text {. }
$$

If we think about $U_{+}$and $U_{-}$as acting on the same space $\mathbb{V}_{T p}$ we can write

$$
U_{+}=U_{-}+2\left\langle\xi_{0}^{+}, n_{0}\right\rangle P_{2}^{*} K P_{2} .
$$

It is not difficult to show that a linear map of a symplectic linear space which takes lagrangian subspaces into lagrangian subspaces must be symplectic up to a scalar multiple and if two linear symplectic operators act on lagrangian subspaces in the same way then they must be equal. Hence the linear map corresponding to the reflection at the boundary must have the block form

where $R=2\left\langle\xi_{0}^{+}, n_{0}\right\rangle P_{2}^{*} K p_{2}$.

$$
\left|\begin{array}{cc}
a I & 0 \\
a R & a I
\end{array}\right|
$$

Taking vectors from $\{0\} \times \mathbb{V}_{p}$ it is easy to establish that actually $a=1$. Finally then

where $s=\tau \sqrt{2 N E}$.

$$
D_{p} T=\left|\begin{array}{cc}
I & s I \\
R & I+s R
\end{array}\right|
$$

For the application in $\S 4$ we will need to know the eigenvalues of $R$ in terms of positions and velocities of the balls. We rescale the velocities by $\sqrt{2 N E}$ so that the phase space velocity in $Q$ has length 1 . Let $\left(q_{1}, \ldots, q_{N}\right), q_{i} \in \mathbb{R}^{d}$ be the positions of the balls. In the neighborhood of a collision of two balls, say the first and the second, we introduce coordinates

$$
z_{1}=\left(q_{1}-q_{2}\right) / \sqrt{2}, \quad z_{2}=\left(q_{1}+q_{2}\right) / \sqrt{2}, \quad z_{i}=q_{i} \text { for } i \geq 3
$$

Now the configuration space $Q$ is described locally by the inequality

$$
\left|z_{1}\right| \geq \sqrt{2} r \text {. }
$$

In these coordinates

$$
\xi_{0}^{+}=\left(V_{\mathrm{rel}} / \sqrt{2}, \bar{v} / \sqrt{2}, v_{3}, \ldots, v_{N}\right)
$$

where $V_{\mathrm{rel}}=v_{1}-v_{2}$ and $\bar{v}=v_{1}+v_{2}$ at the collision and

$$
n_{0}=(e, 0, \ldots, 0) \text {, }
$$


where $e=\left(q_{1}-q_{2}\right) /\left|q_{1}-q_{2}\right|$ at the collision. Hence $w=\left(w_{1}, \ldots, w_{n}\right) \in \mathbb{R}_{T_{p}}$ iff $\left\langle w_{1}, e\right\rangle=$ 0 . The operator $K$ is

$$
\left(w_{1}, \ldots, w_{n}\right) \mapsto\left((\sqrt{2} r)^{-1} w_{1}, 0, \ldots, 0\right) .
$$

Let $y=\left(y_{1}, \ldots, y_{N}\right) \in \mathbb{V}_{T_{p}}$ (i.e. $\left.\left\langle y_{1}, V_{\text {rel }}\right\rangle=0\right)$. Then

$$
P_{2} y=y-\frac{\left\langle y, n_{0}\right\rangle}{\left\langle\xi_{0}^{+}, n_{0}\right\rangle} \xi_{0}^{+} .
$$

Now we can write explicitly the operator

$$
2\left\langle\xi_{0}^{+}, n_{0}\right\rangle P_{2}^{*} K P_{2}=2\left\langle\xi_{0}^{+}, n_{0}\right\rangle K P_{2}-2\left\langle\xi_{0}^{+}, K P_{2} \cdot\right\rangle n_{0}
$$

on $\mathbb{V}_{T p}$.

$$
\left(y_{1}, \ldots, y_{n}\right) \mapsto(r)^{-1}\left(\left\langle V_{\mathrm{rel}}, e\right\rangle y_{1}-\left\langle y_{1}, e\right\rangle\left[V_{\mathrm{ret}}-\frac{\left\langle V_{\mathrm{rel}}, V_{\mathrm{rel}}\right\rangle}{\left\langle V_{\mathrm{rel}}, e\right\rangle} e\right], 0, \ldots, 0\right) .
$$

Hence the nonzero eigenvalues of the last operator are $(r)^{-1} \cos \psi\left|V_{\text {rel }}\right|$ with multiplicity $d-2$ and $(r \cos \psi)^{-1}\left|V_{\text {rel }}\right|$ with multiplicity 1 , where $\psi$ is the angle between $V_{\text {rel }}$ and the line through the centers of the colliding spheres.

Acknowledgements. I gratefully acknowledge many useful discussions I had on the subject of this paper with Anatole Katok and Charles Newman. Part of this work was done while I was visiting the Mathematics Department at Caltech in the summer of ' 85 and I wish to thank them for their hospitality.

This research was supported in part by NSF Grant DMS-8601897.

\section{REFERENCES}

[Ball-Wojt] W. Ballman \& M. Wojtkowski. An estimate for the measure theoretic entropy of geodesic flows. Preprint (1987).

[Ben] G. Benettin. Power law behavior of Lyapunov exponents in some conservative dynamical systems. Physica 13D (1984), 211-220.

[Bun-Sin] L. A. Bunimovich \& Ya. G. Sinai. On a fundamental theorem in the theory of dispersing billiards. Math. USSR, Sb. 19, 3 (1973), 407-423.

[Cher] N. I. Chernov. Construction of transversal fibers in multidimensional semidispersing billiards. Funct. An. Appl. 16 (1982), 35-46.

[Cher-Sin] N. I. Chernov \& Ya. G. Sinai. Entropy of gas of hard spheres with respect to the group of time-space translations. Proceedings of the I. G. Petrovsky Seminar, Vol. 8 (1982), 218-238.

[Kat-Str] A. Katok \& J.-M. Strelcyn. In collaboration with F. Ledrappier \& F. Przytycki. Smooth maps with singularities: Invariant manifolds, entropy and billiards. Lecture Notes in Mathematics, Vol. 1222, Springer: Berlin 1986.

[Led-Str] F. Ledrappier \& J.-M. Strelcyn. A proof of the estimation from below in Pesin's entropy formula. Ergod. Th. \& Dynam. Sys. 2 (1982), 203-219. V. I. Oseledec. The multiplicative ergodic theorem. The Lyapunov characteristic numbers of a dynamical system. Trans. Mosc. Math. Soc. 19, (1968), 197-231.

[Pes] Ya. B. Pesin. Lyapunov characteristics exponents and smooth ergodic theory. Russ. Math. Surveys 32, 4 (1977), 55-114.

[Rue] D. Ruelle. Ergodic theory of differentiable dynamical systems. Publ. Math. IHES 50 (1979), 27-58.

[Sin 1] Ya. G. Sinai. Dynamical systems with elastic reflections. Ergodic properties of dispersing billiards. Russ. Math. Surveys 25, 2 (1970), 137-189.

[Sin 2] Ya. G. Sinai. Entropy per particle for the dynamical system of hard spheres. Preprint, Harvard University (1978). 
[Sin 3] Ya. G. Sinai. Development of Krylov's ideas. Afterword to the Princeton Edition of the book by N. S. Krylov. Works on the Foundations of Statistical Physics. Princeton Series in Physics, Princeton University Press 1979.

[Wojt 1] M. Wojtkowski. Invariant families of cones and Lyapunov exponents. Ergod. Th. \& Dynam. Sys. 5 (1985), 145-161.

[Wojt 2] M. Wojtkowski. Principles for the design of billiards with nonvanishing Lyapunov exponents. Commun. Math. Phys. 105 (1986), 391-441. 